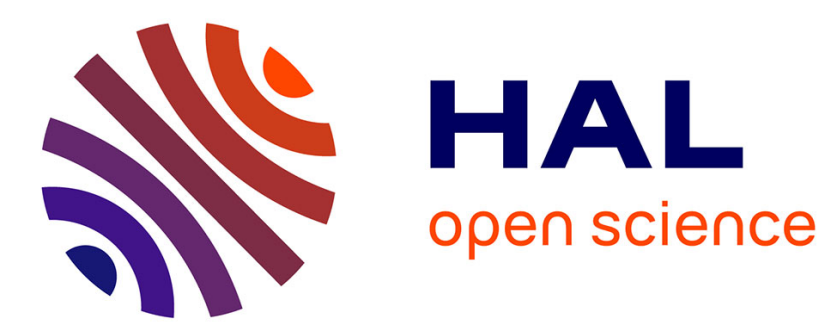

\title{
La musique, c'est du bruit qui pense
}

Alain Vanier

\section{To cite this version:}

Alain Vanier. La musique, c'est du bruit qui pense. Insistance, 2011, Les Voi(x)es de la création, 2

(6), pp.13-21. 10.3917/insi.006.0013 . hal-01524020

\section{HAL Id: hal-01524020 \\ https://hal.science/hal-01524020}

Submitted on 15 Aug 2017

HAL is a multi-disciplinary open access archive for the deposit and dissemination of scientific research documents, whether they are published or not. The documents may come from teaching and research institutions in France or abroad, or from public or private research centers.
L'archive ouverte pluridisciplinaire HAL, est destinée au dépôt et à la diffusion de documents scientifiques de niveau recherche, publiés ou non, émanant des établissements d'enseignement et de recherche français ou étrangers, des laboratoires publics ou privés. 


\section{LA MUSIQUE,}

\section{C'EST DU BRUIT QUI PENSE}

\section{Alain Vanier}

George Bernard Shaw disait qu'un goût prononcé pour un art ne signifiait pas un talent pour celui-ci. Je suis dans ce cas, et, pourtant, je vais vous parler de musique, en toute incompétence donc.

Avez-vous remarqué au concert, ou à l'Opéra, l'importance de la toux dans les moments de silence ? On tousse beaucoup au concert, on a l'impression d'avoir retenu son souffle jusque-là. Une pause entre deux mouvements d'une symphonie, et, d'un seul coup, ce silence est déchiré par des toux que l'on vit souvent comme gênantes ; un moment de relâchement et ces toux qui nous perturbent. C'est ce silence peuplé de bruit que d'habitude nous oublions. Ces toux nous arrachent là où nous étions pour nous faire revenir dans cette salle, quelque chose devient réel soudain. Dans la grande salle de la Philharmonie de Berlin, il y a des petits panneaux qui vous demandent de ne pas tousser, de ne faire aucun bruit étant donné la qualité acoustique de la salle.

Ces toux ne nous dérangent pas le plus souvent au théâtre ou dans un colloque, elles font partie des bruits habituels du silence, nous ne les entendons pas. Mais parfois, la toux prend une autre valeur. Il y a un magnifique concert que Sviatoslav Richter a donné à Sofia en 1958. Ce concertétait retransmis en directà la radio bulgare. Les Tableauxd'une
Alain Vanier, psychanalyste, professeur de psychopathologie clinique, université Paris Diderot, Sorbonne, Paris Cité, CRPMS(EA3522), Paris. 
exposition, un impromptu de Schubert, des études deChopin, de Liszt, etc. Onnecesse pas d'entendrelepublictousser. Or, Richter a refusé de réenregistrer ce concerten studio. Et les toux ajoutent à cet enregistrement une émotion particulière, comme si elles vous introduisaient dans ce lieu même où advient la musique, dontl'enregistrement de studio nous maintient séparé. C'est d'autant plus sensible que Richter, de façon un peu provocante, expliquait qu'à la différence des autres pianistes qui choisissent généralement leur piano sur la qualité duforte, il cherchait l'instrument qui pouvait jouer le plus pianissimo possible, à la limite de l'audible, à la limite du silence. Il insistait sur l'importance des silences en musique qui s'appellent pause, soupirs, demi-pause, etc. Ceux qui ont entendu Richter en concert n'ont pas manqué d'être frappés par ses entrées : il commençait à jouer en même temps qu'il s'asseyait au piano. Sauf pour la Sonate de Liszt, expliquait-il, là, il arrivait, s'installait et comptait dans sa tête jusqu'à 10 ou 20, et, alors seulement jouait le fameux accord. Un compositeur contemporain, JeanYves Bosseur a cette jolie formule : «La musique vient du silence et retourne au silence.»

En musique, le silence sert à séparer des sons, c'est une valeur temporelle. Un « intervalle » est ce qui unit et à la fois sépare, pour reprendre un mot de Jabès. Or le silence n'a pas toujours eu l'importance qu'il a dans la musique d'aujourd'hui. Le culmen est sans doute ce morceau de John Cage 4' 33'. Un pianiste arrive, ferme le couvercle du clavier, puis l'ouvre, sans jouer, au début de chacun des trois mouvements du morceau, dont la durée totale est annoncée par le titre. Vous vous apercevez alors que le silence est peuplé de sons. Le silence en tant que tel n'existe pas, quand bien même nous serions dans un lieu complètement silencieux, le plus silencieux possible, vous entendriez divers bruits de votre corps, bourdonnements d'oreille sans aller jusqu'aux acouphènes ou même hallucinations, il y a toujours quelque bruit defond.

Pour les musicologues, le silence a pris, à partir du xIX ${ }^{e}$ siècle, une valeur particulière. On cite toujours Debussy à ce propos, mais il faut rappeler qu'il y a une sonate de Beethoven qui se termine par une mesure entière de silence. On peut évoquer ce que Benjamin a pu dire sur la notion de critique en opposant l'esthétique gœethéenne à celle du romantisme allemand. Il voit ce mouvement comme une rupture entre une esthétique qui se dit classique, avec l'imitation de la nature, le modèle grec comme Idéal de la production artistique, puis l'advenue du romantisme allemand, avec le passage de l'Idéal àl'Idée et l'introduction de la notion de réflexion. La belle âme - Novalis pour Hegel selon Kojève - qui se consume dans sa propre contemplation est peut-être un peu réductrice quantà la nouveautéqu'apportele romantisme. Les romantiques allemands sont les premiers, après Leibnitz qui a inventé le mot, à s'être interrogés sur ce qu'on pourrait appeler l'inconscienten posant la question de savoir ce qui se passe en chacun malgré soi. Cela fait partie de cette notion de critique dont Benjamin a beaucoup parlé, à savoir l'idée d'une réflexion, d'un retour sur soi-même, d'une interrogation sur le mouvement même de la création qu'implique

\section{INSISTANCE N0 6}


l'idée de critique. Benjamin a montré comment, dans l'avènement du romantisme, s'introduit l'idée d'une critique interne à la production artistique elle-même, une critique lucide de son propre mouvement de production. Ce n'est pas sans rapport avec la mise en évidence d'une coupure, d'une schize pour reprendre le mot de Lacan, qui habite aussi notre rapport à l'écoute, qui habite notre ouïe, notre entendement, en jouant sur le mot. Et cette coupure, le silence - qui prendra une importance de plus en plus grande dans l'écriture musicale - en témoigne, ce qui ne veut pas dire que le silence est la coupure. D'une certaine façon, la musique sert à faire du silence, à faire le silence.

Pour Freud la musique ramène toujours à la parole : « Quiconque veut se donner la peine de porter attention aux airs qu'on fredonne sans en avoir l'intention, souvent sans le remarquer pourra régulièrement mettre à découvert la relation entre les paroles de la chanson et un thème qui occupe l'esprit. » À ce propos, il cite les travaux de Jung et d'autres, mais on se souviendra surtout du livre de Reik, Variations psychanalytiques sur un thème de GustavMahler. Apprenant la mort de son analyste Karl Abraham, une mélodie se met à l'obséder, celle du cinquième mouvement de la deuxième symphonie dite "Résurrection", mouvement qui contient les vers suivants de Klopstock : Aufersteh 'n, ja aufersteh'n wirst du (Tu ressusciteras...). Et tous les écrits de Reik sur la musique ramènent toujours à la parole, au signifiant.

D'ailleurs, Freud met en série le chant avec la citation, le proverbe et même le Witz, le trait d'esprit. Il convient de signaler que l'objection du Witz à la pensée académique est un acte fondamental de l'esthétique du romantisme allemand. Pour Friedrich Schlegel, mais aussi pour Novalis, c'est un quasi concept. Le Witz ne se réduit pas au joke, au trait d'esprit, c'estl'équivoque signifiante qui est mise en avant. De la même manière, le silence dans les écrits même des romantiques allemands prend une place spécifique, ainsi la fonction du fragment, le fait qu'aucun texte ne peut avoir l'ambition de se présenter comme totalisant.

Donc, «la musique, c'est du bruit qui pense », selon la formule de Victor Hugo. Il faut rappeler aussi que c'est récemment que la musique est considérée comme ne faisant pas partie de la pensée. Jusqu'à Descartes, la musique appartenait aux humanités. 
En suivant Freud, peut-on réduire la musique aux paroles ? De la même manière, faut-il ne voir dans toute image qu'un rébus ? C'est notre méthode et cela vaut pour notre travail sur le rêve. Freud dit bien qu'ily a dans le rêve des représentations auditives aussi bien que visuelles. Mais la réduction d'une image à son iconographie ne la résume pas, et un tableau est plus qu'un rébus. Même si nous faisons comme Panofsky, recensant les espèces de plantes représentées dans L'Agneau mystique des Van Eyck avec leurs significations qui rendaient le tableau lisible pour un contemporain, nous n'épuiserons pas la question centrale du tableau.

Nous pouvons appliquer à la musique ce que Lacan dit du tableau. Un tableau, avant tout, permet de se soustraire au regard. On vient y déposer le regard, le regard en tant qu'il est au-dehors. Ilya une schize entre la vision etle regard, entre l'œil et le regard qui est toujours au-dehors. Nous ne nous regardons pas de là où nous voyons. La musique aussi permet de se soustraire à quelque chose, elle permet de se soustraire à la voix qui elle aussi est au-dehors. Les sons y jouent le même rôle que la surface du tableau, un rôle d'écran. L'écran sonore, c'est littéralement un piège à voix ; et dans la musique aussi, on dépose sa voix, ses voix; on dépose la voix comme désir de l'Autre. Mais la musique, comme la peinture, a une double fonction que Freud allouait au théâtre et finalementà toute activité artistique. Àla fois, un effet de libération psychique, un effet de progrès pour le sujet vers quelque chose qui toucherait à sa vérité, et en même temps une consolidation des défenses. L'art a une fonction qui nous amène à un plus de vérité, mais de façon protégée. C'est déjà ce que disait Aristote : la musique a un effet d'apaisement par le moyen de ce qui peut être une transe, dont la forme est variable. La musique vous prend le corps, mais elle ne vous prend le corps que pour vous leurrer, elle ne vous mène pas vers une jouissance qui serait plus authentique ou plus vraie. Ce n'est pas ça, mais quand même. D'où le complément nécessaire de la drogue par exemple. Il en faut plus puisqu'il s'agit d'atteindre une jouissance qui, par définition, est inatteignable, et même n'existe pas. Cette jouissance-là est tout aussi limitée que toute autre.

Normalement, nos voix sont méconnues, à moins que nous soyons psychotiques. Les voix qui nous habitent sont élidées à l'état de veille. Nous les remisons dans un lieu qui est celui del'Autre. Lacan dità un moment donnéà ses auditeurs que, quoiqu'il dise, ils nel'entendent pas, car la seule chose qu'ils entendent ce sont leurs voix. Nous sommes tous occupés chacun par nos voix. Etc'està travers nos voix que nous entendons éventuellement quelque chose de l'autre. On ne les entend pas, comme d'ailleurs, dans l'état de veille, on ne voit pas le regard. Mais nous sommes sous le regard, comme nous sommes sous la voix, avec ce que ça peut avoir de contraignant, de cadrant, de limitant pour chacun au quotidien.

La voix, comme le regard, est virée au compte de l'Autre, mais pas dans la psychose. L'halluciné n'est pas dans la dépendance d'un objet au champ de l'Autre, c'est là sa liberté. Dans l'hallucination acoustico-verbale, ce sont des phrases tronquées que les hallucinés entendent. Ce 
sont des phrases à compléter. C'est sensible chez Schreber. L'énoncé se présente toujours en deux morceaux. Un névrosé, c'est quelqu'un qui peut se parler, s'interpeller, sans que ça le divise réellement. Dans la psychose, cette coupure-là, symbolique dans la névrose, devient réelle.

Cette dimension est quelque chose que les psychiatres classiques avaient observée: les hallucinés font des mouvements des lèvres. Dans la vie normale, quand on parle, on ne s'entend pas parler. Il y a une élision. Dire de quelqu'un «il s'écoute parler» est péjoratif. Il est vrai que s'il s'écoute parler, il ne vous parle plus. Nous oublions que nous sommes les premiers auditeurs de notre parole. C'est d'ailleurs un exercice assez compliqué pour les chanteurs puisqu'on nes'entend pas chanter comme les autres vous entendent.

Pour Lacan, ces fragments de jouissance, le regard et la voix sont des figures du surmoi. Or le surmoi, originairement, « ça n'est rien que la grosse voix », ce qui renvoie au surmoi précoce de Melanie Klein. Une grosse voix qui n'a aucune autorité. Ce qui fait sa puissance tientà son potentiel de jouissance parce qu'elle manifeste un autre qui apparaît alors comme réel. Après coup, l'œEdipe va refouler, habiller cette voix, lui donnant un certain nombre de coordonnées, la phallicisant, c'est-à-dire la séparant, en permettant cette articulation symbolique et le refoulement. Or, cette voix - cet objet $a$ - séparée, détachée, a une fonction très particulière, elle est le témoin de ce lieu de l'Autre à qui je la remets continûment. C'estsa normativation par l'CEdipe. C'est très exactement ce que dit Freud dans les Trois Essais sur la théorie sexuelle. Mais la voix au sens de la psychanalysen'est pas la sonorité. Ce n'est pas le timbre, ni l'intonation, ni ce qui resterait quand on a retiré la parole, ni la pure image acoustique. Pour Lacan, ce qui s'en approche le plus, c'est la scansion, soit la coupure même. Elle est ce qui choit de la parole, mais ce n'est pas ce reliquat de timbre ou quoi que ce soit d'autre de sa matérialité. Elle est ce qui choit de la parole, c'est-à-dire littéralement un vide, un silence, qui pourrait être le pur silence, un vide au cœur de tout objet, celui de l'objet $a$, c'est-à-dire un trou. Il y a ce son si particulier, bitonal, émis par le Shofar, le jour de Yom Kippour, soufflé dans la corne qui évoque le bélier sacrifié. Ce moment est, selon Lacan, «un moment de résolution del'angoisse». Toutela 
journée de Kippour, le sujet a été confronté à la question de la faute et du pardon, de la culpabilité, dans une dimension où le désir de l'Autre prend la forme d'un commandement. Or précisément, la voix, c'estle désir del'Autre; désir de l'Autre et non désir à l'Autre, puisque Lacan distingue la voix et le regard. Ce moment de résolution, ce son qui vient sur le silence du commandement et qui soulage de l'impératif de la parole, évoque ce qu'Alain Didier-Weill avait écrit sur « La note bleue», nommée ainsi par Delacroix à propos d'un morceau de Chopin. Je l'ai toujours identifié à la fin du Prélude ${ }^{\circ} 4$ de Chopin. Chacun la sienne peut-être, il y en a plusieurs, mais, en tout cas, à cet endroit-là, il pourraity avoir un silence.

Sionpoursuitl'analogie avecla peinture, pensez au petit pan de mur jaune de la Recherche. Quand on voit la vue de Delft de Vermeer, on le cherche partout. Celui que j'ai vu ne coïncide pas avec la représentation que je m'en étais faite. Je pensais que c'était une tache qui venait obturer le point de fuite dans la construction du tableau. Pas du tout. Il est dans une position quelconque. Il est plutôt légèrement à droite, n'a aucune fonction; c'est une petite tache jaune qui, si elle n'était pas délimitée, si elle n'occupait pas une certaine place dans l'architecture du tableau serait une pure tache en tant que telle. Elle n'a pas de fonction obturante par rapport à ce qui serait un point de fuite ou un point qui troueraitl'espace dans la construction même du tableau.

Dans tout le tableau, il y a une absence, un trou et un écran. Dans la lecture que Lacan fait des Menines de Vélasquez, il voit ce trou sous les jupes de l'Infante. Les trous dans ce tableau, ce ne sont pas les fenêtres, judicieusement masquées, ni le point de fuite, encadré par une porte. Ce sont des trous leurrants dans la construction de l'image. Le point très précis qui centre complètement le tableau, c'estexactement « la fente » de l'Infante voilée derrière ses jupes, et son corrélat, le tableau que peint Velasquez et qu'on ne voit pas. Ce qu'il y a à voir, on ne le voit pas. C'est du même ordre que la note bleue; c'est un moment de résolution, il montre et masque, protège à la fois. La note bleue, ce sontlesjupes, cen'est pas la fente. La note bleue aussi est un point de concours, de résolution des diverses lignes qui composent la musique entre système mélodique et harmonie. Boulez disait d'ailleurs que le grand musicien est celui qui fait passer une nouvelle forme de diagonale ou de construction entre les deux axes de la musique : l'axe mélodique, contrapuntique, horizontal, etl'axe harmonique, vertical. De Bach à Beethoven, on va du contrepoint à l'harmonie, et avec Mahler, c'est le retour du contrepoint. Dans un livre récent d'Alex Ross, The Rest is Noise, la musique du Xx ${ }^{\mathrm{e}}$ siècle commence avec Mahler, à juste titre donc.

L'art nous permet d'approcher impunément - de façonapaisée pour reprendre le motd'Aristotela limite propre au désir, désir dont résolument nous nous maintenons à distance. L'art fait voir le point de visée du désir et en même temps il l'écrante. Le beau est à la fois un aveuglement, et, en même temps, quelque chose qui pointe l'horreur. Et d'ailleurs, « en finir avec le beau » est un des mots de Lacan, mais aussi, une caractéristique des arts contemporains. C'est 
pourquoi il n'y a pas d'esthétique de la psychanalyse. C'est ce qu'ont amorcé les romantiques paradoxalement : faire apparaître cette béance au cœur de l'œuvre, au cœur de l'être même qui se réfléchit.

Si on revient à cet agencement horizontal et vertical qui caractérise l'écriture musicale, entre harmonie et mélodie, on peut reprendre ce que Deleuze et Guattari font de cette organisation qui renvoie aussià l'agencement paradigme et syntagme chez Saussure. Pour eux, c'est la base de ce qu'ils appellent un «système ponctuel » qui est constitutif d'un «bloc sonore » pour la musique, et qui peut parfois se réduire à un point. Ils donnent comme exemple le «si» de Berg dans Wozzeck, le « la » de Schumann, etc. Mais ce qui est frappant en les lisant est l'absence de toute référence au silence.

« Un enfant dans le noir, saisi par la peur, se rassure en chantonnant. » C'est le premier aspect, où la chanson « est un saut du chaos à un début d'ordre dans le chaos ». Deuxième aspect: chez soi, dans son lieu, on a tracé un cercle, un cercle de sons : le bruit de la radio, les chansons qu'on fredonne marquent les limites du territoire. Les maisons, les lieux que nous habitons sont sonores. $0 \mathrm{n}$ voit bien à quel point la fonction de ce cercle est essentielle puisque quand on entend la radio ou la télévision du voisin, on peut être exaspéré. Ou encore, à certains moments, on entrouvre le cercle, pas du côté du chaos préexistant, mais d'un côté plus sécurisé et, écrivent-ils, « on sort de chez soi au fil d'une chansonnette ». Ces trois aspects sont ceux de " la ritournelle ». La ritournelle a une fonction de territorialisation. Et par exemple, ils proposent une lecture originale du Fort-Da qui, selon eux, fonctionne comme une ritournelle. Ce n'est pas simplement une opposition signifiante, Fort-Da, qui vient symboliser quelque chose, mais c'est aussi une fonction de la ritournelle. $0 r$, il faudrait ajouter que cette ritournelle-là est posée, elle aussi, sur une béance, celle de l'absence de la mère et de sa voix. C'estle silence de la mère que le Fort-Da maîtrise, symbolise et leurre. Aucun retour de la mère ne permettra à la retrouvaille de s'équivaloir à la trouvaille. La retrouvaille est toujours marquée d'un manque fondamental, d'un vide. Donc, au cœur de tout objet sonore, au cœur du bloc sonore aussi.

La fente de l'Infante, tout comme le silence, sont toujours voilés. La musique fabrique donc le silence en tant qu'il n'existe pas. S'il existait, 
le silence, ce serait la voix même. Mais le silence qui n'existe pas est gros de l'imminence de la voix. Et c'est làle point où s'arrête le parallèle avec le regard. Dans le champ scopique, le sujet, rappelle Lacan, est déterminé par la séparation, comme coupure de l'objet $a$, coupure que constitue l'objet $a$. Le regard introduitquelque chose de fascinatoire. La capture imaginaire qu'il provoque, l'assigne, le détermine et nous détermine dans le champ du visible. Ainsi la construction de la perspective dans le tableau assigne une place précise au spectateur. Par contre, dans le champ invoquant, le sujet est indéterminé. La première rencontre avec le désir de l'Autre, avec cette question que pose la bête à tête de chameau dans la nouvelle de Cazotte, Le diable amoureux - que veux-tu ? che vuoi ?ne procure aucune détermination du sujet. À cela, le sujet ne peut répondre qu'en se faisant objet. Voilà d'ailleurs pourquoi en clinique, l'hallucination visuelle et l'hallucination acoustico-verbale n'ont pas la même valeur.

La musique n'a-t-elle pour fonction que de donner ses coordonnées au silence, voire de l'encadrer, et en même temps de le faire éclater? Or, il n'y a pas un mot dans Deleuze et Guattari sur le silence, malgré le schizophrène venant comme modèle, lui dont le discours est marqué par les barrages, les moments de fading, les interruptions, précisément tous ces silences qui viennent, d'une façon surprenante ou déroutante pour l'interlocuteur, arrêter le discours. Pour Deleuze et Guattari, la ritournelle n'est pas de la musique mais elle constitue le cœur de toute musique. Ils donnent comme exemple le fameux «Ah vous dirais-je maman » de Mozart. Ainsi « la ritournelle est à la fois le contenu de la musique et aussi le moyen de conjurer la musique ", écrivent-ils, qui « a soif de destruction ». Car la musique arrache la ritournelle à la territorialité mais elle ne l'élimine pas. La musique déterritorialise la ritournelle. On pourrait dire qu'elle l'arrache et la conserve en même temps en son sein, elle la relie à la répétition. Mais elle fait aussi apparaître cette dimension d'écran; d'où la nécessité du silence. On peut penser ici à la notion de séparation comme deuxième opération de la réalisation du sujet après l'aliénation. Car dans ce moment de séparation, après le moment où le sujet s'aliène au signifiant, il y a un temps de rencontre du manque dans l'Autre, au cœur même de la parole de l'Autre, sans oublier que toute parole adressée à un enfant peut prendre valeur d'intimation. Elle fonctionne comme un commandement. Mais ce qui est décisif pour l'enfant dans ce moment-là, c'est précisément ce que l'Autre ne dit pas. Ce qui est décisif, ce sont précisément les silences, les intervalles du discours qui font que «l'enfant peut se poser la question : il me dit ça, mais qu'est-ce qu'il veut? qu'est-ce qu'il ne dit pas ? » Désir inarticulable qui joue dans l'intervalle entre les signifiants, dans les silences de la parole. Or, il n'y a pas d'accès direct à cette béance car la séparation, inévitablement, rate etfait toujours retour à l'aliénation. Ce silence oul'imminence du désir de l'Autre, voilà l'angoisse. L'angoisse que le phobique traite par la peur, car le phobique a cette expérience particulière comparable à la toux qu'on entend soudain au concert quand la musique fait silence. Dans le silence de la 
chambre, la nuit, il entend les craquements du bois, le frôlement du vent, il a peur. Il a peur de quelque chose qui serait le signe de l'Autre, non pas son signifiant mais le signe de l'Autre, de sa présence, l'imminence de la voix, l'imminence de son désir.

$\mathrm{Si}$, pour reprendre le mot de Deleuze et Guattari, la musique déterritorialise la ritournelle, elle la reterritorialise en même temps. Voilà peut-être pourquoi Freud a pu écrire à propos de Hans : «Depuis le début de l'anxiété, [il] montre un intérêt accru pour la musique et développe ses dons musicaux héréditaires ", peut-être pourdompter le silence, pour le cadrer. Ainsi, la musique produit-elle le silence, ou plutôt fait-elle apparaitre le silence comme peuplé de bruits, ce silence impossible qu'il n'y a pas, elle rend troublante les bruits du silence, les sons du silence, elle les rend inhabituels, étrangement inquiétants, réels, comme la construction du phobique. Elle nous fait pressentir l'impossible du silence absolu.

Résumé : "La musique vient du silence et retourne au silence " écrit JeanYves Bosseur. N'est-ce pas la façon dont elle élève « l'objet à la dignité de la Chose »?

Mots-clés : musique, objet a, silence, voix.

Summary: " Music comes from silence and goes back to silence », wrote JeanYves Bosseur. Isn't it one way to raise « the object to the dignity of the Thing " ?

Keywords : Music, object a, silence, voice.

Resumen : "La música viene del silencio y vuelve al silencio », escribió Jean-Yves Bosseur. ¿No es eso lo que eleva el « objeto a la dignidad de la Cosa »?

Palabras clave : Música, objeto a, silencio, voz. 